\title{
Measuring the local gravitational field using survival resonances in a dissipatively driven atom-optics system
}

\author{
Shijie Chai, Julia Fekete, and Mikkel F. Andersen* \\ The Dodd-Walls Centre for Photonics and Quantum Technologies, Department of Physics, University of Otago, Dunedin 9054, New Zealand
}

(Received 23 August 2018; revised manuscript received 19 October 2018; published 10 December 2018)

\begin{abstract}
We do a proof-of-principle demonstration of an atomic gravimeter based on survival resonances of dissipatively driven atoms. Exposing laser-cooled atoms to a sequence of near-resonant standing-wave light pulses reveals survival resonances when the standing-wave interference pattern accelerates. The resonant accelerations determine the local gravitational acceleration and we achieve a precision of $5 \mathrm{ppm}$ with a drop distance less than $1 \mathrm{~mm}$. The incisiveness of the resonances scales with the square of the drop time. Present results indicate that an appropriately designed atomic gravimeter based on survival resonances might be able to reach a precision of $1 \mu \mathrm{Gal}$ with a 10 -cm-high fountain. The relatively simple experimental construction of this technique may be of interest for a compact absolute atomic gravimeter.
\end{abstract}

DOI: 10.1103/PhysRevA.98.063614

\section{INTRODUCTION}

Atom interferometry has provided a variety of techniques for precision measurements [1-3] with impressive accuracies for both fundamental research and practical applications. Light-pulse atom interferometers exploit atom-light interactions to coherently manipulate matter waves [1,2]. Laser pulses can act as refractive, reflective, and absorptive elements for matter waves. The interference phenomena arising from splitting and recombining matter waves has enabled precision measurements of rotation [4,5], gravity gradients [6], the Newtonian gravitational constant $[7,8]$, and the fine-structure constant $[9,10]$. The high performance makes it an ideal tool for testing fundamental laws of physics [11-14]. Among the many applications of atom interferometry, atomic gravimeters [15-20] are of great attraction for their range of practical applications in geophysics, cosmology, and metrology.

The atom optics $\delta$-kicked rotor (AODKR) $[21,22]$ provides a protocol for carrying out interferometric measurements of the gravitational acceleration $g$ [23-28]. In recent work, we showed that a variant of it displays a quantum interference phenomenon deemed "survival resonances" [29]. They arise when spatially periodic dissipation or loss is added to the temporally periodic kicks in the standard $\delta$-kicked rotor. Consequently, the atom number is not a conserved quantity and a meaningful dynamic observable is the survival probability of the atoms. Varying the pulse interval reveals a series of survival resonances when it is an integer multiple of half the Talbot time. These resonances are a result of matter-wave Talbot-Lau interference [30,31].

In this work, we investigate an atomic gravimeter that exploits survival resonances [11,29-31]. To sense the gravitational field, the atoms interact with pulses of a vertically arranged standing wave (parallel to the local gravitational field).

\footnotetext{
*mikkel.andersen@otago.ac.nz; http://www.physics.otago.ac.nz/nx/mikkel/home-page.html
}

Gravity eliminates the survival resonances since it adds extra phases during the free evolution between light pulses. Nevertheless, linearly sweeping the frequency difference between the two counterpropagating light waves forming the standing wave, effectively accelerates the standing-wave interference pattern such that it co-moves with the free falling atoms. This leads to reemergence of the survival resonances, from which we can deduce the local $g$ value. This approach offers several technical simplicities with respect to present atomic gravimeters [15-20]. (i) The frequency of the standing-wave light is close to the atomic transitions, which enables us to derive it from the same laser source used for cooling and trapping atoms. This allows for an easier construction since less or simpler laser systems are required. (ii) The Talbot-Laufacilitated interferometric survival resonances allow for their observation using a thermal atomic cloud effectively without an unwanted background. This reduces the complexity of the setup by avoiding the need for cooling atoms beyond standard polarization gradient cooling. (iii) The experimental output is a simple internal state detection, similar to the Raman-based gravimeter $[15,17]$. The simple implementation makes it an attractive candidate for a compact atomic gravimeter.

The structure of this paper is as follows. Section II provides a description of the experimental system. In Sec. III we give a theoretical description of the survival resonances in the presence of the gravitational field with a four-level model that considers the possible atomic transitions on the $D_{2}$ line of rubidium atoms. In Sec. IV, we utilize the four-level model to revisit survival resonances without gravitational effects and study the phase modulation caused by a standing wave with finite detuning. Section $\mathrm{V}$ shows the emergence of survival resonances when the acceleration of the standing wave is swept. Section VI gives the dependence of the height and width of the resonant peak on different experimental parameters. For an appropriate parameter combination, Sec. VII demonstrates a local gravitational acceleration measurement reaching a precision of $5 \mathrm{ppm}$ by interrogating over a drop distance about $1 \mathrm{~mm}$. It also discusses the potential limitation 


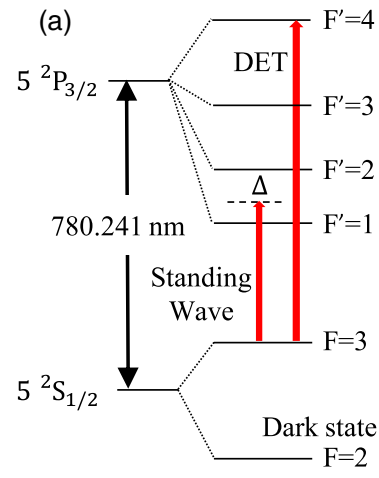

(b)

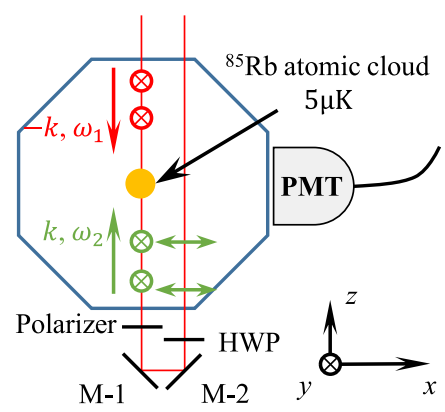

(c)
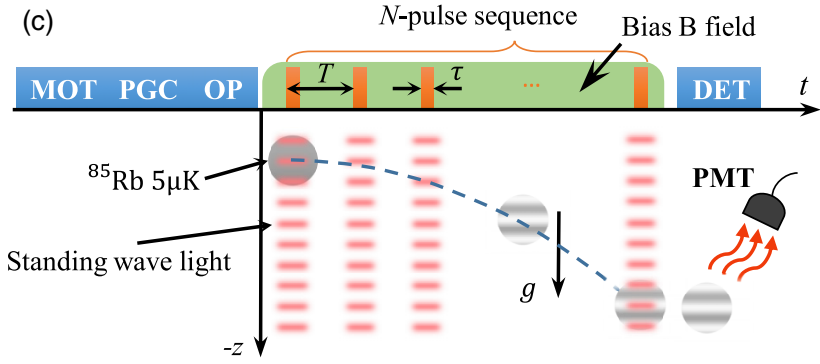

FIG. 1. (a) ${ }^{85} \mathrm{Rb} D_{2}$ transition hyperfine structure (not to scale). (b) Simplified schematic of the vertically arranged standing-wave light beam. The double arrows and dots represent the polarizations of the laser beams. (c) Time sequence of the experiment (not to scale). Acronyms: MOT magneto-optical trap; PGC polarization gradient cooling; OP optical pumping; DET atomic state detection; and PMT photomultiplier tube.

and application of the technique. The paper concludes with Sec. VIII.

\section{EXPERIMENTAL SYSTEM}

In the experiment, we use ${ }^{85} \mathrm{Rb}$ atoms and their two hyperfine ground states $F=3$ and $F=2$ as the initial state and a "dark" state, respectively [see the atomic level diagram for the $D_{2}$ line in Fig. 1(a)]. The $F=2$ ground state is dark to both the standing wave and detection beam and we consider the atom to be lost from the system once it goes to this state. The standing-wave light is tuned close to the open atomic transition from the $F=3$ ground state to the $F^{\prime}=2$ excited hyperfine state. The detuning is $\Delta / 2 \pi=-10 \mathrm{MHz}$ except in measurements where the detuning is the parameter varied. When exposed to such a standing-wave light beam, the atoms will scatter photons and be optically pumped out of the initial ground state at the antinodes, while they remain at the nodes. The standing-wave light therefore forms an "absorption grating" that spatially modulates the amplitude of the atomic wave function. Additionally, the finite detuning leads to the formation of a spatially periodic dipole potential that will modulate the phase of the atom. The standing-wave light beam thereby acts as a hybrid of an amplitude and a phase grating [29].

Figure 1(b) shows how the standing wave forms due to the interference between two laser beams (with opposite wave vectors) counterpropagating in the science chamber. To achieve a stringent rejection of relative phase noise between these two components, we deliver them with orthogonal polarizations through the same single-mode polarization maintaining fiber. The output light is collimated to have a Gaussian profile with a diameter of $7.5 \mathrm{~mm}\left(1 / e^{2}\right)$ and then split by a high polarization extinction ratio Wollaston prism. As shown in Fig. 1(b), beam 1 with frequency $\omega_{1}$ and polarization along the $y$ axis is aligned vertically and centered at the atomic ensemble. The other beam with frequency $\omega_{2}$ initially propagates parallel to beam 1 and is then reflected by two silver-coated polarization maintaining mirrors $(M-1$ and $M-2)$ such that it counterpropagates beam 1 at the position of the atoms. Its polarization is rotated 90 degrees by a half-wave plate (HWP) and further cleaned by a polarizer. This ensures that the two beams have the same linear polarization for high contrast interference. Additionally, having the same light intensity for each beam (typically $15 \mathrm{~mW} / \mathrm{cm}^{2}$ ) maximizes the standing-wave contrast. By linearly ramping the frequency difference between the two beams, the standing-wave interference pattern can move with a constant acceleration along the vertical $z$ axis.

As the timeline in Fig. 1(c) shows, we start each of the experimental cycles by trapping a cloud of ${ }^{85} \mathrm{Rb}$ atoms from a thermal atomic beam in a three-dimensional (3D) magneto-optical trap (MOT). By increasing the detuning while reducing the optical power of the cooling beams and simultaneously switching off the quadrupole magnetic field, we cool the atoms to $5 \mu \mathrm{K}$ by polarization gradient cooling (PGC). The repump beam maintains its optical intensity during the PGC process to ensure all the atoms are optically pumped into the $F=3$ hyperfine ground state. After the state preparation, we turn off the cooling and repump beams and expose the atoms to the standing-wave pulse sequence. The period of the sequence is $T$ and the duration for each pulse is $\tau$, where $\tau=400 \mathrm{~ns}$ is sufficiently short so that it fulfills the Raman-Nath condition. $N$ equals to 5 throughout the paper unless otherwise indicated. A bias magnetic field of $5.6 \mathrm{G}$ is switched on along the $x$ axis (perpendicular to both the propagation and polarization directions of the standing wave beam) to quench dark states in the $F=3$ manifold. Immediately after the standing-wave pulse sequence, we use a photomultiplier tube (PMT) to record the survival probability (the proportion of the atoms remaining in the $F=3$ ground state) through detecting the fluorescence signal when exciting the atoms on the closed transition from $F=3$ to $F^{\prime}=4$ [see the long red arrow in Fig. 1(a)]. The measurements are averaged over 20 experimental runs and the error bar in figures denotes the standard deviation of the mean unless stated in the caption.

\section{THEORY}

We consider the vertical standing-wave arrangement shown in Fig. 1(b). We need to include the gravitational potential $M g z$ ( $M$ is the mass of atom) and the time-dependent standing wave which can move spatially to match the free falling frame of the atoms. Furthermore, the two-level atomic model in [29] did not capture all features of the resonance peaks. To improve this, we use a four-level atomic model that contains all the possible atomic transitions on the $D_{2}$ line. In this section, we give the theoretical description of the survival 
probability with the four-level model in the presence of the gravitational field.

\section{A. System Hamiltonian}

The four levels we consider in the ${ }^{85} \mathrm{Rb}$ atom consist of one hyperfine ground state $F=3$ denoted $|3\rangle$, and three excited hyperfine states $|e\rangle$ with $F^{\prime}=2, F^{\prime}=3$, and $F^{\prime}=4$ on the $D_{2}$ line [see Fig 1(a)]. The time evolution of the atomic wave function is governed by the Hamiltonian

$$
\begin{aligned}
H= & \frac{p^{2}}{2 M}+M g z+\sum_{e=2}^{4}\left(\hbar \omega_{e}-\frac{i \hbar \Gamma}{2}\right)|e\rangle\langle e| \\
& -\mathbf{d} \cdot \mathbf{E} \times \sum_{n^{\prime}=0}^{N} \Pi\left(t-n^{\prime} T, \tau\right),
\end{aligned}
$$

where we ignore the atom-atom interaction, which is valid for a dilute sample. $z$ and $p$ are the atomic position and momentum operators along the $z$ axis (parallel to the local gravitational acceleration $g$ ). $N$ is the number of pulses applied to the atoms. We choose the $F=3$ ground state energy to be zero and use $\hbar \omega_{e}$ to represent the atomic energies for the three excited states. The imaginary term $-i \hbar \Gamma / 2$ describes the relaxation of the excited states to the $F=2$ dark ground state, which causes the loss of atoms. In the Hamiltonian, we ignore the spontaneous decay back to the $F=3$ initial ground state which will lead to an incoherent background in the experiments. We account for this by manually adding an offset to calculations based on Eq. (1) when comparing to the experimental data. The atom-light interaction is described by using dipole approximation, where $\mathbf{d}$ is the electric dipole moment and $\mathbf{E}$ is the electric field of the standing-wave light beam. We shape the standing-wave pulses as a series of tophat functions

$$
\Pi\left(t^{\prime}, \tau\right)= \begin{cases}1 & 0 \leqslant t^{\prime} \leqslant \tau, \\ 0 & t^{\prime}<0 \text { and } t^{\prime}>\tau,\end{cases}
$$

where $\tau$ is the pulse duration.

As Fig. 1(b) shows, the standing-wave light field is formed by the two counterpropagating laser beams: beam 1 (downwards propagating with wave vector $-k$ ) and beam 2 (upwards propagating with wave vector $k$ ), where we assume the standing wave is exactly parallel to the $z$ axis. When the pulse is on, the electric field $\mathbf{E}$ has the form

$$
\mathbf{E}(z, t)=\frac{1}{2} \hat{\varepsilon}_{1} E_{1} e^{i\left(-k z-\omega_{1} t\right)}+\frac{1}{2} \hat{\varepsilon}_{2} E_{2} e^{i\left(k z-\omega_{2} t\right)}+\text { c.c. },
$$

where $\hat{\varepsilon}_{1}$ and $\hat{\varepsilon}_{2}$ denote the polarization vectors for the laser beams 1 and 2 . The two laser beams have parallel linear polarization so that $\hat{\varepsilon}_{1}=\hat{\varepsilon}_{2} . E_{1}$ and $E_{2}$ are the electric field amplitudes and c.c. means the complex conjugate. $\omega_{1}$ and $\omega_{2}$ are the optical angular frequencies for the laser beams 1 and 2. By linearly ramping the optical frequencies of the two beams with a same constant rate $\dot{\omega}=a_{\text {eff }} k$ and opposite sign, $\omega_{1}=\omega_{0}+\dot{\omega} t$ and $\omega_{2}=\omega_{0}-\dot{\omega} t$, we are able to accelerate the standing-wave interference pattern with an effective acceleration $a_{\text {eff }}$. We start with a static standing wave by having the same frequency $\omega_{0}$ for the two beams, and initiate its acceleration when switching off the optical pumping beams, which is the repump light in this case. For simplicity, we consider the standing-wave light field to have perfect contrast by fulfilling the conditions: $E_{1}=E_{2} . \mathbf{E}(z, t)$ in Eq. 3 is therefore

$$
\begin{aligned}
\mathbf{E}(z, t)= & \hat{\varepsilon}_{1} E_{1} \cos \left(-k z-\omega_{0} t-\frac{1}{2} a_{\mathrm{eff}} k t^{2}\right) \\
& +\hat{\varepsilon}_{1} E_{1} \cos \left(k z-\omega_{0} t+\frac{1}{2} a_{\mathrm{eff}} k t^{2}\right) \\
= & 2 \hat{\varepsilon}_{1} E_{1} \cos \left(k z+\frac{1}{2} a_{\mathrm{eff}} k t^{2}\right) \cos \left(\omega_{0} t\right) .
\end{aligned}
$$

Using Eq. (4) in Eq. (1), transferring $H$ to a rotating frame that rotates at the light frequency $\omega_{0}$, and adapting the rotating wave approximation reduce $H$ to

$$
\begin{aligned}
H= & \frac{p^{2}}{2 M}+M g z+\sum_{e=2}^{4}\left[-\left(\hbar \Delta_{3 e}+\frac{i \hbar \Gamma}{2}\right)|e\rangle\langle e|\right. \\
& +\frac{\hbar \Omega_{3 e}}{2} \cos \left(k z+\frac{1}{2} a_{\mathrm{eff}} k t^{2}\right) \times\left(\sum_{n^{\prime}=0}^{N} \Pi\left(t-n^{\prime} T, \tau\right)\right) \\
& \times(|e\rangle\langle 3|+| 3\rangle\langle e|)]
\end{aligned}
$$

where $\Delta_{3 e}$ denotes the detuning of the standing-wave light with respect to the three atomic transitions, and $\Omega_{3 e}$ represents the maximum on-resonance Rabi frequency for these transitions at the anti-nodes of the standing wave.

\section{B. Gauge transformations}

The Hamiltonian $H$ in Eq. (5) gives the full description of the system. In principle, one can use it to compute all the physical quantities that are of interest. Nevertheless, it is desirable to simplify the calculations by transforming $H$ to a convenient frame. We first translate the wave function to a frame that moves at the standing-wave acceleration in position space $|\widetilde{\psi}\rangle=\mathcal{T}^{\dagger}|\psi\rangle[$ where $\mathcal{T}(\Delta z)=\exp (i \Delta z p / \hbar)$ is the translation operator and $\left.\Delta z=a_{\mathrm{eff}} t^{2} / 2\right]$, and the transformed Hamiltonian $\widetilde{H}$ that governs the evolution of $|\widetilde{\psi}\rangle$ is $\widetilde{H}=\mathcal{T}^{\dagger} H \mathcal{T}+a_{\mathrm{eff}} t p$

$$
\begin{aligned}
\widetilde{H}= & \frac{p^{2}}{2 M}+M g\left(z-\frac{1}{2} a_{\mathrm{eff}} t^{2}\right)+a_{\mathrm{eff}} p t \\
& +\sum_{e=2}^{4}\left[-\left(\hbar \Delta_{3 e}+\frac{i \hbar \Gamma}{2}\right)|e\rangle\langle e|+\frac{\hbar \Omega_{3 e}}{2} \cos (k z)\right. \\
& \left.\times\left(\sum_{n^{\prime}=0}^{N} \Pi\left(t-n^{\prime} T, \tau\right)\right)(|e\rangle\langle 3|+| 3\rangle\langle e|)\right] .
\end{aligned}
$$

Now the standing wave is stationary. However, the system is not spatially periodic due to the $M g z$ term. To further simplify $\widetilde{H}$, we apply another gauge transformation: $\mathcal{U}(\Delta p)=$ $\exp (-i z \Delta p / \hbar)$ with $\Delta p=M g t$. The gauge-transformed 
atomic state $|\tilde{\tilde{\psi}}\rangle=\mathcal{U}^{\dagger}|\widetilde{\psi}\rangle$ is then governed by $\tilde{\tilde{H}}=\mathcal{U}^{\dagger} \tilde{H} \mathcal{U}-$ $M g z$ [32]:

$$
\begin{aligned}
\tilde{H}= & \frac{p^{2}}{2 M}-\left(g-a_{\mathrm{eff}}\right) p t+\frac{M g}{2}\left(g-3 a_{\mathrm{eff}}\right) t^{2} \\
& +\sum_{e=2}^{4}\left[-\left(\hbar \Delta_{3 e}+\frac{i \hbar \Gamma}{2}\right)|e\rangle\langle e|+\frac{\hbar \Omega_{3 e}}{2} \cos (k z)\right. \\
& \left.\times\left(\sum_{n^{\prime}=0}^{N} \Pi\left(t-n^{\prime} T, \tau\right)\right)(|e\rangle\langle 3|+| 3\rangle\langle e|)\right]
\end{aligned}
$$

After these two consecutive gauge transformations, we now have a system that is spatially periodic, and the position of the standing-wave pattern is time-independent. This comes at the expense of having explicit time-dependence in $\tilde{\tilde{H}}$. The free evolution when the standing wave is off is governed by the Hamiltonian $\tilde{\tilde{H}}_{\text {free }}=\frac{p^{2}}{2 M}-\left(g-a_{\text {eff }}\right) p t$, where we neglect the term $\frac{M g}{2}\left(g-3 a_{\text {eff }}\right) t^{2}$ that does not have physical significance. Equation (7) is preferable for computation since the free evolution Hamiltonian only contains $p$ and not $z$, which implies that we can perform efficient time evolution in momentum space. On the other hand, if we invoke the Raman-Nath approximation during a pulse, the atom-light interaction only depends on the position $z$, which again indicates we can expeditiously carry out the computation in position space. The fast Fourier-Transform (FFT) and inverse fast Fourier-Transform (IFFT) allow us to transfer the atomic states between position and momentum spaces efficiently, which ensures we can numerically simulate the dynamics of the system quickly.

\section{Time evolution}

Due to the top-hat nature of our standing-wave pulse sequence, we can divide the time evolution governed by Eq. (7) into two segments: the dynamics during the standing-wave pulse, and the free dynamics between pulses. By computing the time evolution of the two situations separately, we can find the pulse-to-pulse time evolution operator as their product.

\section{Grating operator, dynamics during the pulse}

During the standing-wave light pulse, the Hamiltonian $\tilde{\tilde{H}}$ in Eq. (7) reduces to:

$$
\begin{aligned}
\tilde{\tilde{H}}_{\text {pulse }}= & \sum_{e=2}^{4}\left[-\left(\hbar \Delta_{3 e}+\frac{i \hbar \Gamma}{2}\right)|e\rangle\langle e|\right. \\
& \left.+\frac{\hbar \Omega_{3 e}}{2} \cos (k z)(|e\rangle\langle 3|+| 3\rangle\langle e|)\right],
\end{aligned}
$$

where we omitted the atomic motion since the pulse duration $\tau$ is short (Raman-Nath approximation). The time evolution of the atomic wave function $|\tilde{\psi}\rangle$ is given by

$$
|\tilde{\tilde{\psi}}, \tau\rangle=\exp \left(-\frac{i}{\hbar} \tilde{\tilde{H}}_{\text {pulse }} \tau\right)|\tilde{\psi}, 0\rangle .
$$

As discussed before, we are only interested in the atoms that remain in their initial $F=3$ ground state. Equation (9) will leave the atoms in a superposition of the different internal states. However, we can define a "grating operator" $\tilde{\tilde{G}}$ that describes the effect of the standing-wave pulse on the wave function of the atoms that remain in $F=3$ state as

$$
\tilde{\tilde{G}}=\left\langle 3\left|\exp \left(-\frac{i}{\hbar} \tilde{\tilde{H}}_{\text {pulse }} \tau\right)\right| 3\right\rangle,
$$

where $\tilde{\tilde{G}}$ only operates on atoms in the $F=3$ ground state. Note that $\tilde{\tilde{G}}$ is a nonunitary operator and the probability for the atom surviving in the $F=3$ state after one pulse can be calculated as $\left\langle\tilde{\tilde{\psi}}_{3}\left|\tilde{\tilde{G}}^{\dagger} \tilde{\tilde{G}}_{\mid}\right| \tilde{\tilde{\psi}}_{3}\right\rangle$, where the subscript 3 indicates the wave function is for atoms in the $F=3$ ground state. Importantly, because the gauge-transformed standing-wave pattern does not move spatially, we only need to calculate the grating operator once.

\section{Dynamics between standing wave pulses}

We now study the dynamics between pulses, when the atom undergoes free evolution that is governed by the Hamiltonian $\tilde{\tilde{H}}_{\text {free }}=p^{2} / 2 M-\left(g-a_{\text {eff }}\right) p t$. Note $\tilde{\tilde{H}}_{\text {free }}$ is explicitly time dependent, but it commutes with itself at different times, which enables us to construct a time-evolution operator $\tilde{\tilde{F}}=$ $\exp \left(-\frac{i}{\hbar} \int_{t_{1}}^{t_{2}} \tilde{\tilde{H}}_{\text {free }} d t\right)$ from $t_{1}$ to $t_{2}$. Because of the time dependence of the Hamiltonian, the time-evolution operator will differ for different free space time intervals. In particular, the time-evolution operator $\tilde{\tilde{F}}_{n^{\prime}}$ from $t=\left(n^{\prime}-1\right) T$ to $t=n^{\prime} T$ becomes

$$
\begin{aligned}
\tilde{F}_{n^{\prime}}= & \exp \left(-\frac{i}{\hbar} \frac{p^{2}}{2 M} T\right) \\
& \times \exp \left(\frac{i}{\hbar}\left[\frac{p\left(g-a_{\mathrm{eff}}\right)}{2}\left(2 n^{\prime}-1\right)\right] T^{2}\right) .
\end{aligned}
$$

The first term arises from the atomic kinetic energy. The second term incorporates the effect of gravity. When the acceleration of the standing wave $a_{\text {eff }}$ matches $g$, it becomes the identity operator and the system becomes equivalent to one where the atoms receive pulses horizontally [29].

\section{N-pulse train}

We can now obtain an expression for the time-evolution operator after an $N$-pulse train. Taking advantage of the temporal periodicity of the pulse sequence, we can construct a pulseto-pulse evolution operator $\tilde{\tilde{U}}_{n^{\prime}}=\tilde{\tilde{F}}_{n^{\prime}} \tilde{\tilde{G}}$, from $t=\left(n^{\prime}-1\right) T$ to $t=n^{\prime} T$. Recall that $\tilde{\tilde{F}}_{n^{\prime}}$ does not change the internal state of the atoms so $\tilde{\tilde{U}}_{n^{\prime}}$ gives the dynamics of the wave function of the atoms that remain in the $F=3$ initial ground state. After receiving $N$ pulses, the atomic wave function $\left|\tilde{\tilde{\psi}}_{3}, N T\right\rangle$ is given by

$$
\left|\tilde{\tilde{\psi}}_{3}, N T\right\rangle=\tilde{\tilde{U}}_{N} \tilde{\tilde{U}}_{N-1} \cdots \tilde{\tilde{U}}_{1}\left|\tilde{\tilde{\psi}}_{3}, 0\right\rangle .
$$

Since $\tilde{\tilde{G}}$ is nonunitary, so is this evolution. Equation (12) allows for the computation of the survival probability of atoms in the $F=3$ ground state, which can be measured experimentally by state detection. Note there is no need for transferring $\left|\tilde{\tilde{\psi}}_{3}, N T\right\rangle$ back to its initial frame before computing the survival probability. The gauge transforms are unitary so they do not change the survival probability. 

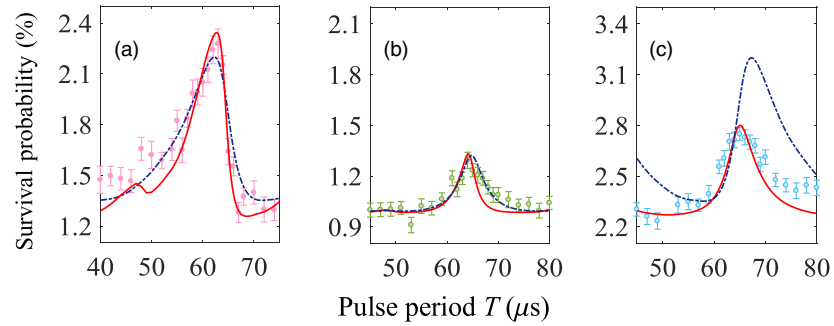

FIG. 2. The measured (markers with error bars) and calculated (lines) survival resonances around $T=T_{\mathrm{T}}$ after a $N=7, \tau=300 \mathrm{~ns}$ pulse sequence. The incoming light intensity is $9 \mathrm{~mW} / \mathrm{cm}^{2}$. The detunings $\Delta$ are (a) $-2 \pi \times 3 \mathrm{MHz}$, (b) $0 \mathrm{MHz}$, and (c) $2 \pi \times 3 \mathrm{MHz}$. The red solid (or dark blue dot-dashed) lines are calculations based on the four-level (or two-level) model.

\section{Numerical calculations}

To calculate the survival probability of a thermal ensemble of atoms, we propagate an incoherent set of initially Gaussian wave packets using Eq. (12). The width of the Gaussian wave packets is determined by the temperature of the atomic cloud and the initial positions of the ensemble of wave packets cover a period of the standing wave homogeneously. We operate with $\tilde{\widetilde{G}}$ in position space where it is diagonal, and then evolve the wave function between pulses in momentum space where $\tilde{\tilde{F}}_{n^{\prime}}$ is diagonal. FFT and IFFT rapidly transfer the atomic wave function between these two spaces making the computation efficient. Finally, the survival probability compared to experiments will be averaged over the initial ensemble.

\section{SURVIVAL RESONANCES EFFECTIVELY WITHOUT THE GRAVITATIONAL FIELD}

We now revisit the survival resonances investigated in Ref. [29] by using the more advanced four-level model. We study the system in the absence of the gravitational field by implementing the standing wave either horizontally or vertically and setting $a_{\text {eff }}=g$.

\section{A. Resonant pulse period}

Figure 2 displays the survival probability as a function of the pulse period $T$ for a $N=7$ pulse train, where the standing wave is formed by retroreflecting a horizontal laser beam. It shows survival resonance peaks near the Talbot time $T_{\mathrm{T}}$ for different laser detunings (where $T_{\mathrm{T}}=\pi M / \hbar k^{2}=64.8 \mu \mathrm{s}$ is the Talbot time for ${ }^{85} \mathrm{Rb}$ atoms [29]). The markers with error bars are measured data and the lines are the numerical calculation based on Eq. (12). The emergence of the survival resonances can be conceptually understood through the matter-wave Talbot-Lau effect (see Fig. 3) [29-31]. In Fig. 3, we consider an incoherent atomic wave impinging on two identical pure absorption gratings that are separated by a time $T=T_{\mathrm{T}} / 2$. The matter-wave density distribution will form an interference pattern downstream. At time $t=2 \times T_{\mathrm{T}} / 2$, the pattern becomes a near perfect "self-image" of the grating itself, with the peak distribution aligned with the slits of the grating (the nodes of the standing wave). Additionally, the matter-wave Talbot-Lau effect ensures the formation of the self-image when the grating separation is any integer

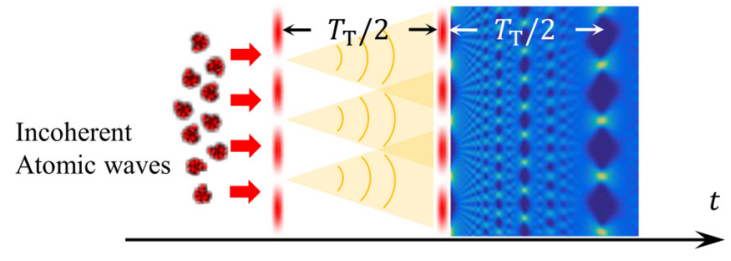

FIG. 3. The matter-wave Talbot-Lau effect. The color code represents the atomic density. The vertical axis is the coordinate along the standing wave, and the horizontal axis is time. When impinging an incoherent atomic wave onto two identical gratings that are separated by a time $T_{\mathrm{T}} / 2$, a self-image forms at time $T_{\mathrm{T}} / 2$ after the second grating.

multiples of half the Talbot time $T=n T_{\mathrm{T}} / 2($ where $n \in \mathbb{N}$ ) [29]. If a third standing-wave pulse is applied when these self-images form, the atoms have a high probability of survival since they are localized around the nodes. Furthermore, when there are more than three pulses, the previous two will ensure that the atoms are near the nodes of the standing wave whenever $T=n T_{\mathrm{T}} / 2$. This yields the high survival around $T=T_{\mathrm{T}}$ shown in Fig. 2. For finite detunings, the presence of the dipole potential gives rise to a microlensing effect that enhances the peak height and are responsible for the asymmetry of the peak, for example, seen in Fig. 2(a) [29].

The lines in Fig. 2 are the numerical calculations based on Eq. (12), with the blue dot-dashed lines for a two-level model that only includes the excited state closest to resonance with the standing wave light, and the red lines are for the four-level model discussed in Sec. III. For the experimental data we use a horizontal retroreflected laser beam to form the standing wave and account for the imbalanced intensity of the standing-wave light using the measured losses from the view ports and the retromirror. Recall, we also add an offset to the calculated curve to compensate the incoherent background due to spontaneous decay back to the $F=3$ ground state. The four-level model agrees with the measurements without any fitted parameters.

Treating the atom as a two-level system by only including one excited state in the calculation of $\tilde{\tilde{G}}$ (the $F^{\prime}=2$ state in Fig. 2), also gives reasonable agreements when the standing-wave light intensity is fitted. The fitted intensity agrees with the measurement within $25 \%$. However, we see that the two-level model fails to capture the asymmetry in the peak height for red and blue detuning clearly visible in the experimental data. The asymmetry arises because the other excited hyperfine states will alter the phase and amplitude modulations differently on opposite sides of resonance. All following calculations exclusively use the four-level model without any fitting parameters since it captures the data best.

\section{B. Long pulse periods}

Figure 4 shows four survival resonance peaks with long pulse intervals, where we use a vertical standing-wave beam arrangement and set $a_{\text {eff }}=g$. In this case, the system behaves identically to the one where there are no gravitational effects [see Eq. (11)]. The peaks are at the 15th, 30th, 45th, and 60th Talbot time $T_{\mathrm{T}}$, which shows that this matter-wave interferometric phenomenon persists for large pulse periods. For $T$ 


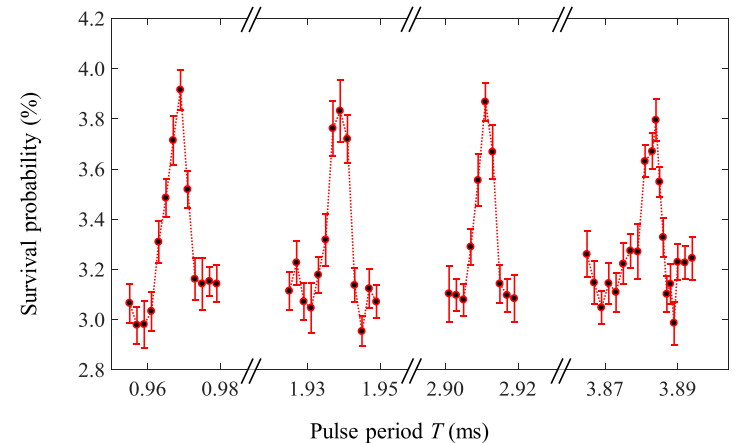

FIG. 4. A comb of survival resonances at $T=$ 15th, 30th, 45th, and 60th $T_{\mathrm{T}}$, with a vertically arranged standing wave where $a_{\mathrm{eff}}=g$.

longer than $4 \mathrm{~ms}$, the atoms drop out of the detection region of the PMT.

Figure 4 shows a slow decay of the peak height when the pulse period is extended. The experimental effects that can contribute to this could be: low-frequency phase noise of the standing wave due to vibrations of the reflecting mirrors, transverse thermal motion of the atoms in the standing-wave beam that could contain phase distortions, and small residual forces on the atoms, for example, due to stray magnetic field gradients. Finally, the atoms being split to higher diffraction orders will leave the detection area faster than the atoms that rapidly go to the dark state, which will also yield a monotonic decay of the signal size with the increasing pulse period.

\section{SURVIVAL RESONANCES WITH GRAVITATIONAL FIELD}

The survival resonances can be used to make a measurement of the local gravitational acceleration $g$. We can conceptually explain this as follows: when the evolution between pulses includes gravity, the self-image (see Fig. 3) does not align with subsequent gratings since the atoms accelerate. Nevertheless, by moving the standing-wave interference pattern spatially, such that it matches the self-image, the survival resonance re-emerges. Equation (11) displays this since the second exponential term will suppress survival when $a_{\text {eff }} \neq g$, while it becomes the identity operator when $a_{\mathrm{eff}}=g$.

Figure 5 displays two data series where $a_{\text {eff }}$ is swept while keeping $T=0.969 \mathrm{~ms}\left(15 \mathrm{th} T_{\mathrm{T}}\right)$ or $T=1.941 \mathrm{~ms}\left(30\right.$ th $T_{\mathrm{T}}$ ). We again see survival resonances. In both data sets a peak appears when $a_{\text {eff }}$ matches $g$. The black lines are fits with a sum of five Gaussian functions that share peak height, width, and offset. However, the peak position of each Gaussian is a free parameter. The peak position of the central Gaussian yields the local $g$ value.

An interesting feature of the resonances shown in Fig. 5 is that we observe survival resonances not only when $a_{\text {eff }}=g$ but also for $a_{\text {eff }}=g+j(\lambda / 2) / T^{2}$, where $j \in \mathbb{Z}$ and $\lambda / 2$ is the spatial periodicity of the standing wave. To understand this, we consider the initial thermal atomic cloud as an incoherent sum of plane waves. The spatial periodicity of the standing wave in combination with the Bloch's theorem ensures the atomic state originating from a plane-wave $\left|p_{0}\right\rangle$

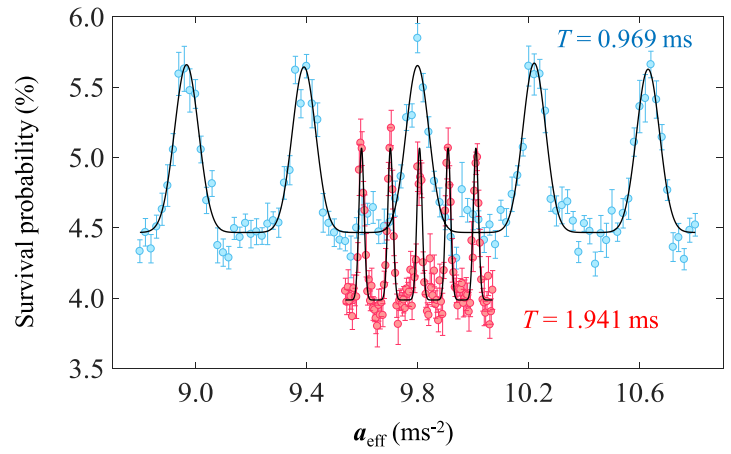

FIG. 5. The measured survival probability as a function of the effective acceleration $a_{\text {eff }}$, with the pulse period $T=0.969 \mathrm{~ms}$ (blue) and $1.941 \mathrm{~ms}$ (red). The solid black lines are the fitting curves with five Gaussian peaks.

to have the form of $\sum_{m} c_{m}(t)\left|p_{0}+2 m \hbar k\right\rangle$, where $p_{0}$ is the initial momentum and $c_{m}(t)$ denotes the time-dependent amplitude of the $m$ th diffraction order. Applying the free evolution operator $\tilde{\tilde{F}}_{n^{\prime}}$ in Eq. (11) on this state for $T=T_{\mathrm{T}} / 2$ yields the final state with the form of $\sum_{m} c_{m}\left[t=\left(n^{\prime}-\right.\right.$ 1)T] $\exp \left[-i\left(m^{2} \pi+\left(p_{0}+j \hbar k\right) m \pi / \hbar k\right)\right]\left|p_{0}+2 m \hbar k\right\rangle$,

where we omitted a global phase. From this we see that the effect of the integer $j$ is equivalent to a shift of the initial momentum $p_{0}$ with amount of $j \hbar k$. However, since the survival probability is averaged over a broad initial momentum distribution, there is no net effect from shifting it and a survival resonance equivalent to $j=0$ emerge for all $j \in \mathbb{Z}$.

\section{RESONANCE'S DEPENDENCE ON EXPERIMENTAL PARAMETERS}

A leading motivation of this work is to understand the behavior of survival resonances as a function of $a_{\mathrm{eff}}$ with different experimental parameters. To this end, we performed a series of experiments by studying the incisiveness and height of the resonances as a function of several variables. Note that we acquire the resonance peaks as a function of $a_{\text {eff }}$ by using the specific $T$ values that allow for an observable survival resonance. Figures 6, 7, and 8 show the width (and height

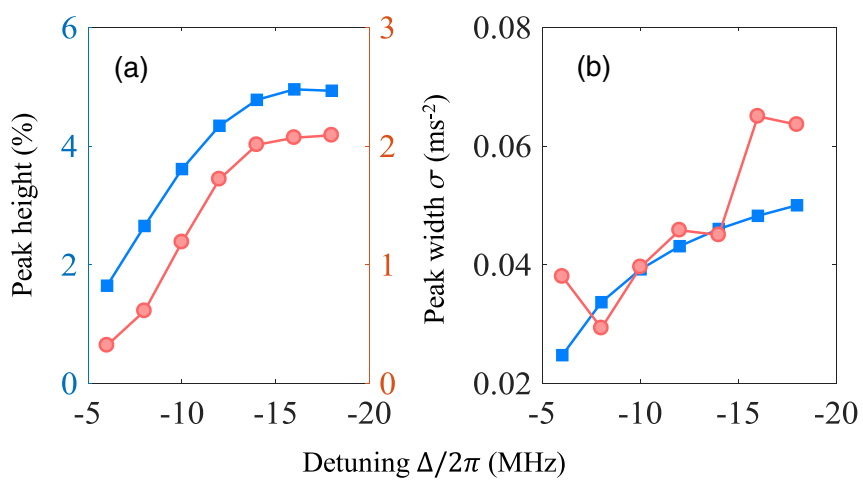

FIG. 6. Measured (red circles) and calculated (blue squares) (a) peak height and (b) peak width (standard deviation $\sigma$ ) as a function of the laser detuning $\Delta$, at $T=15 T_{\mathrm{T}}$. 


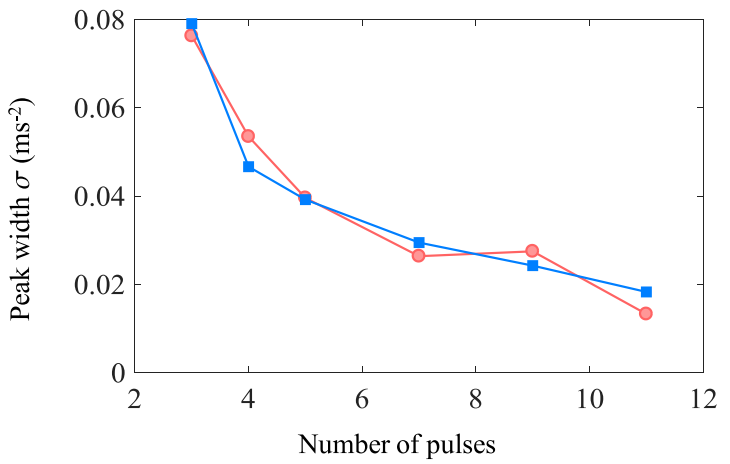

FIG. 7. Measured (red circles) and calculated (blue squares) peak width as a function of the pulse number $N$, with $T=15 T_{\mathrm{T}}$.

in one case) as a function of different parameters, where the width and height are obtained by fitting a Gaussian to the measured (or calculated) data around the central peak shown in Fig. 5.

\section{A. Strength of interaction}

When the strength of the interaction between the atoms and the standing-wave light increases the resonant peak as a function of $a_{\text {eff }}$ lose height, but becomes narrower. This effect can, for example, be seen in Fig. 6 for changing the detuning. Similar data for varying the standing-wave light intensity shows the same trends. Figure 6 shows that the height [Fig. 6(a)] and width [Fig. 6(b)] grows as the detuning of the standing-wave light is increased. When the detuning exceeds $14 \mathrm{MHz}$, the light scattering rate at the antinodes of the standing wave is insufficient to remove all atoms and the growth in the peak height ceases. If the detuning is increased further the system approaches a standard AODKR and the standing wave is not dissipative. The atoms thereby survive regardless of their position and the survival resonances vanish. In Fig. 6(a) the trends of the experimental data and the calculation agree but the experimental data shows a smaller peak height. This is because we use relatively long pulse separations, and as we saw in Fig. 4, the data display a decay of peak height that is not captured by the calculation.

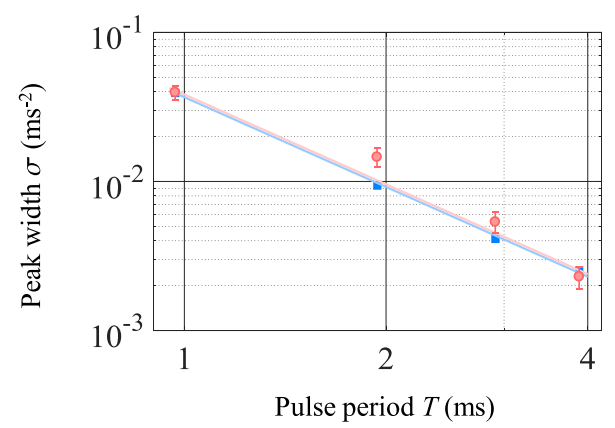

FIG. 8. Measured (red circles) and calculated (blue squares) peak width as a function of pulse period $T$ in a double-log scale. Error bars denote the confidence interval for one standard deviation for the Gaussian fitting. The blue (or red) curve is a scaling law to the calculation (or measurement) with a fixed power of -2 .

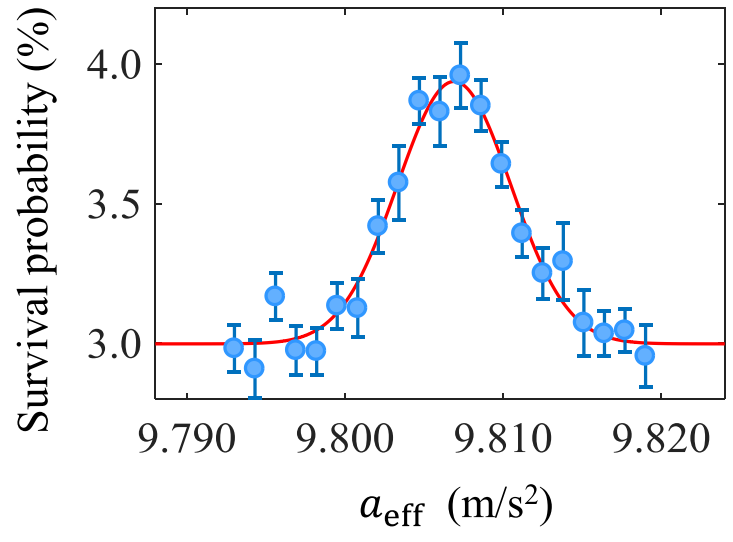

FIG. 9. A survival resonance peak against $a_{\mathrm{eff}}$ that is similar to Fig. 5 but with $T=3.882 \mathrm{~ms}$. The red line is a Gaussian fit for extracting the $g$ value.

\section{B. Number of pulses}

In Fig. 7, both the experiment and calculation show that the peak width becomes narrower when the number of standing wave pulses $(N)$ increase. This monotonic trend occurs since a higher $N$ causes a population of higher diffraction orders that accumulate phase at higher rate during the free evolution. Higher $N$ therefore requires $a_{\text {eff }}$ to be close to $g$ to guarantee that different diffraction orders are in phase at the application of the subsequent pulses. Furthermore, increasing $N$ also increases the total interrogation time. Increasing $N$ decreases the peak height (data not shown) since there is a finite probability for light scattering even when close to a standing-wave node.

\section{Pulse period}

Figure 8 displays the measured (red circles) and calculated (blue squares) peak width when varying the pulse period $T$ from the 15th to the 60th Talbot time. The monotonic decrease can be understood from Eq. (11). The second term, which is responsible for the peak when $a_{\text {eff }}$ is scanned, contains the product of $g-a_{\mathrm{eff}}$ and $T^{2}$. The width should therefore display a scaling law as a function of $T$ with the power of -2 . The solid lines in Fig. 8 are fits to the calculation (blue curve) and measurement (red curve) with a $T^{-2}$ law. We see that the fitted curves capture the data reasonably well. Classical physics conceptually explains the $T^{-2}$ dependence since the position of a free falling atom is proportional to the square of the fall time.

\section{MEASUREMENT OF $\boldsymbol{g}$ AND DISCUSSION}

While our present apparatus is not optimized for precision measurements it is interesting to estimate if it is feasible to reach a precision in the $\mu \mathrm{Gal}$ regime which would make the technique of interest for geoscience applications. So we carry out a measurement of $g$ and discuss how it can be improved.

\section{A. Measurement of $g$}

To measure $g$ we first map out the survival resonance using $T=3.882 \mathrm{~ms}\left(60^{\text {th }} T_{\mathrm{T}}\right)$. Figure 9 displays the measured data and we fit it with a Gaussian function. We then employ a 
standard protocol for performing high-rate measurement by taking data on the maximal slopes on each side of the fringe [15]. About 1400 repetitions yields a $g$ value of 980710(5) $\mathrm{mGal}$ (corrected for a present angle of $\theta=3.3$ mrad between the standing wave and the direction of the gravitational force).

\section{B. Discussion}

The atomic gravimeter presented in this paper shows an uncertainty of $\Delta g / g=5 \times 10^{-6}$. It is feasible to achieve a higher precision by implementing the following alterations. Using a $10-\mathrm{cm}$ fountain geometry instead of a fall distance about $1 \mathrm{~mm}$ will lead to a 300 -fold reduction of the uncertainty due to the narrowing of the peak with $T$. By implementing atomic shot-noise-limited detection and increasing the atom number from the present $\sim 10^{5}$ to above $10^{6}$ should give further improvement of more than an order of magnitude. It might therefore be possible to reach a precision in the $\mu \mathrm{Gal}$ regime.

Since we use near resonant light for the standing wave, the presence of the atoms affects the wavelength of the standingwave light by changing the index of refraction [33]. This effect is presently negligible compared to our uncertainty, but in improved measurements it will need to be considered. Similar to other kinds of atomic gravimeters, the effects of Coriolis force, gravity gradients, wavefront distortions and so on, will also impact the results and need to be corrected in a similar manner as done in [15].

The easy implementation of the system also makes it attractive for applications with less requirement to the precision. For example, determine the first digits of $g$ for standard high precision atomic gravimeters [15-20], or measure the free fall of antimatter [34].

\section{SUMMARY}

We study the survival resonances in an atom-optics system that is driven by temporally and spatially periodic dissipa- tion. The emergence of such resonances arises due to the matter-wave Talbot-Lau effect. Modeling the internal states of the atoms as a four-level system that includes the relevant transitions on the $D_{2}$ line gives excellent agreement between experiments and calculations, without any fitting parameters, for short time intervals between pulses. For longer time intervals we observe a reduction in the peak height that is not captured by the model, while it still captures the peak width well. Using a vertical standing wave we observe survival resonances when sweeping the acceleration of the standing-wave interference pattern. These resonances allow us to determine the local gravitational acceleration, and we characterize their dependence on the experimental parameters. Our present results indicate that a properly designed system can reach a precision in the $\mu \mathrm{Gal}$ regime with integration times less than 10 min.

The advantages of utilizing survival resonances for an atomic gravimeter are the simplicity of the system. Unlike driving Raman transition or Bragg transition, our standingwave light is near resonant, and can therefore be derived simply from the same laser used for cooling the atoms. The strong atom-light interaction of near-resonant light also fulfills the low power requirement. Since the survival resonances are facilitated by the matter-wave Talbot-Lau effect, they yield a clear signal, effectively without an unwanted background, using a thermal atomic source. Finally, the simple internal state detection and the ability to recycle the atoms from previous runs will help to increase the repetition rate. Therefore, the techniques discussed here may be of interest for a compact absolute atomic gravimeter or applications with less stringent requirement to the precision.

\section{ACKNOWLEDGMENTS}

We wish to thank Eric $\mathrm{Xu}$ for assistance with collecting data. This work was supported by the NZ-MBIE (Contract No. UOOX1402).
[1] P. R. Berman, Atom Interferometry (Academic, New York, 1996).

[2] A. D. Cronin, J. Schmiedmayer, and D. E. Pritchard, Optics and interferometry with atoms and molecules, Rev. Mod. Phys. 81, 1051 (2009).

[3] C. L. Degen, F. Reinhard, and P. Cappellaro, Quantum sensing, Rev. Mod. Phys. 89, 035002 (2017).

[4] T. L. Gustavson, P. Bouyer, and M. A. Kasevich, Precision Rotation Measurements with an Atom Interferometer Gyroscope, Phys. Rev. Lett. 78, 2046 (1997).

[5] D. S. Durfee, Y. K. Shaham, and M. A. Kasevich, Long-Term Stability of an Area-Reversible Atom-Interferometer Sagnac Gyroscope, Phys. Rev. Lett. 97, 240801 (2006).

[6] J. M. McGuirk, G. T. Foster, J. B. Fixler, M. J. Snadden, and M. A. Kasevich, Sensitive absolute-gravity gradiometry using atom interferometry, Phys. Rev. A 65, 033608 (2002).

[7] J. B. Fixler, G. T. Foster, J. M. McGuirk, and M. A. Kasevich, Atom interferometer measurement of the newtonian constant of gravity, Science 315, 74 (2007).
[8] G. Rosi, F. Sorrentino, L. Cacciapuoti, M. Prevedelli, and G. M. Tino, Precision measurement of the Newtonian gravitational constant using cold atoms, Nature (London) 510, 518 (2014).

[9] R. Bouchendira, P. Cladé, S. Guellati-Khélifa, F. Nez, and F. Biraben, New Determination of the Fine Structure Constant and Test of the Quantum Electrodynamics, Phys. Rev. Lett. 106, 080801 (2011).

[10] R. H. Parker, C. Yu, W. Zhong, B. Estey, and H. Müller, Measurement of the fine-structure constant as a test of the Standard Model, Science 360, 191 (2018).

[11] S. Fray, C. Alvarez Diez, T. W. Hänsch, and M. Weitz, Atomic Interferometer with Amplitude Gratings of Light and Its Applications to Atom Based Tests of the Equivalence Principle, Phys. Rev. Lett. 93, 240404 (2004).

[12] L. Zhou, S. Long, B. Tang, X. Chen, F. Gao, W. Peng, W. Duan, J. Zhong, Z. Xiong, J. Wang, Y. Zhang, and M. Zhan, Test of Equivalence Principle at $10^{-8}$ Level by a Dual-Species DoubleDiffraction Raman Atom Interferometer, Phys. Rev. Lett. 115, 013004 (2015). 
[13] P. W. Graham, J. M. Hogan, M. A. Kasevich, and S. Rajendran, New Method for Gravitational Wave Detection with Atomic Sensors, Phys. Rev. Lett. 110, 171102 (2013).

[14] T. Kovachy, P. Asenbaum, C. Overstreet, C. A. Donnelly, S. M. Dickerson, A. Sugarbaker, J. M. Hogan, and M. A. Kasevich, Quantum superposition at the half-metre scale, Nature (London) 528, 530 (2015).

[15] A. Peters, K. Y. Chung, and S. Chu, Measurement of gravitational acceleration by dropping atoms, Nature (London) $\mathbf{4 0 0}$, 849 (1999); High-precision gravity measurements using atom interferometry, Metrologia 38, 25 (2001).

[16] A. Louchet-Chauvet, T. Farah, Q. Bodart, A. Clairon, A. Landragin, S. Merlet, and F. Pereira Dos Santos, The influence of transverse motion within an atomic gravimeter, New J. Phys. 13, 065025 (2011).

[17] Z.-K. Hu, B.-L. Sun, X.-C. Duan, M.-K. Zhou, L.-L. Chen, S. Zhan, Q.-Z. Zhang, and J. Luo, Demonstration of an ultrahighsensitivity atom-interferometry absolute gravimeter, Phys. Rev. A 88, 043610 (2013).

[18] P. A. Altin, M. T. Johnsson, V. Negnevitsky, G. R. Dennis, R. P. Anderson, J. E. Debs, S. S. Szigeti, K. S. Hardman, S. Bennetts, G. D. McDonald, L. D. Turner, J. D. Close, and N. P. Robins, Precision atomic gravimeter based on Bragg diffraction, New J. Phys. 15, 023009 (2013).

[19] T. Mazzoni, X. Zhang, R. Del Aguila, L. Salvi, N. Poli, and G. M. Tino, Large-momentum-transfer Bragg interferometer with strontium atoms, Phys. Rev. A 92, 053619 (2015).

[20] K. S. Hardman, P. J. Everitt, G. D. McDonald, P. Manju, P. B. Wigley, M. A. Sooriyabandara, C. C. N. Kuhn, J. E. Debs, J. D. Close, and N. P. Robins, Simultaneous Precision Gravimetry and Magnetic Gradiometry with a Bose-Einstein Condensate: A High Precision, Quantum Sensor, Phys. Rev. Lett. 117, 138501 (2016).

[21] F. L. Moore, J. C. Robinson, C. Bharucha, P. E. Williams, and M. G. Raizen, Observation of Dynamical Localization in Atomic Momentum Transfer: A New Testing Ground for Quantum Chaos, Phys. Rev. Lett. 73, 2974 (1994).

[22] F. L. Moore, J. C. Robinson, C. F. Bharucha, B. Sundaram, and M. G. Raizen, Atom Optics Realization of the Quantum $\delta$-Kicked Rotor, Phys. Rev. Lett. 75, 4598 (1995).
[23] G. Behinaein, V. Ramareddy, P. Ahmadi, and G. S. Summy, Exploring the Phase Space of the Quantum $\delta$-Kicked Accelerator, Phys. Rev. Lett. 97, 244101 (2006).

[24] R. K. Shrestha, S. Wimberger, J. Ni, W. K. Lam, and G. S. Summy, Fidelity of the quantum $\delta$-kicked accelerator, Phys. Rev. E 87, 020902(R) (2013).

[25] R. A. Horne, R. H. Leonard, and C. A. Sackett, Utility of atomic kicked-rotor interferometers for precision measurements, Phys. Rev. A 83, 063613 (2011).

[26] B. Daszuta and M. F. Andersen, Atom interferometry using $\delta$ kicked and finite-duration pulse sequences, Phys. Rev. A 86, 043604 (2012).

[27] I. Talukdar, R. Shrestha, and G. S. Summy, Sub-Fourier Characteristics of a $\delta$-Kicked-Rotor Resonance, Phys. Rev. Lett. 105, 054103 (2010).

[28] R. Dubertrand, I. Guarneri, and S. Wimberger, Fidelity for kicked atoms with gravity near a quantum resonance, Phys. Rev. E 85, 036205 (2012).

[29] S. Chai, J. Fekete, P. McDowall, S. Coop, T. Lindballe, and M. F. Andersen, Survival resonances in an atom-optics system driven by temporally and spatially periodic dissipation, Phys. Rev. A 97, 033616 (2018).

[30] A. Turlapov, A. Tonyushkin, and T. Sleator, Optical mask for laser-cooled atoms, Phys. Rev. A 68, 023408 (2003).

[31] A. Turlapov, A. Tonyushkin, and T. Sleator, Talbot-Lau effect for atomic de Broglie waves manipulated with light, Phys. Rev. A 71, 043612 (2005).

[32] R. Bach, K. Burnett, M. B. d'Arcy, and S. A. Gardiner, Quantum-mechanical cumulant dynamics near stable periodic orbits in phase space: Application to the classical-like dynamics of quantum accelerator modes, Phys. Rev. A 71, 033417 (2005)

[33] G. K. Campbell, E. Leanhardt, J. Mun, M. Boyd, E. W. Streed, W. Ketterle, and D. E. Pritchard, Photon Recoil Momentum in Dispersive Media, Phys. Rev. Lett. 94, 170403 (2005).

[34] P. Hamilton, A. Zhmoginov, F. Robicheaux, J. Fajans, J. S. Wurtele, and H. Müller, Antimatter Interferometry for Gravity Measurements, Phys. Rev. Lett. 112, 121102 (2014). 